\title{
Investigation of Photonic Band Gap in Si-Based One-Dimensional Photonic Crystal
}

\author{
Kulandaisamy S. Joseph Wilson, Vasan Revathy \\ Department of Physics, Arul Anandar College (Autonomous) Karumathur, Madurai, India \\ Email: wilsonpra@yahoo.co.in
}

Received September 23, 2013; revised October 25, 2013; accepted November 18, 2013

Copyright (C) 2013 Kulandaisamy S. Joseph Wilson, Vasan Revathy. This is an open access article distributed under the Creative Commons Attribution License, which permits unrestricted use, distribution, and reproduction in any medium, provided the original work is properly cited. In accordance of the Creative Commons Attribution License all Copyrights (C) 2013 are reserved for SCIRP and the owner of the intellectual property Kulandaisamy S. Joseph Wilson, Vasan Revathy. All Copyright (C) 2013 are guarded by law and by SCIRP as a guardian.

\begin{abstract}
A one-dimensional silicon based photonic crystal with nonlinear defect layers is examined. The linear and nonlinear optical properties are analyzed. The transmission spectrum was obtained by applying the optical transfer matrix formalism to the photonic crystal. The various transmittance peaks obtained with the defect layers are investigated. The nature of the transmittance peak is analyzed with the number of defect layers.
\end{abstract}

Keywords: Photonic Band Gap; 1D Photonic Crystal; $\mathrm{Si}-\mathrm{SiO}_{2}$; Transmittance

\section{Introduction}

The concept of photonic crystals (PCs) was first proposed by Yablonovitch and John in 1987 [1,2], who found that using periodic arrangement of dielectric materials enables us to control the propagation of electromagnetic waves. The most significant feature of a PC is the existence of photonic band gap (PBG), a frequency range in which the electromagnetic waves can not propagate. The PBG in a PC is analogous to the electronic band gap in a solid because of the similarity between the structural periodicity of a $\mathrm{PC}$ and the periodic potential energy in a solid. If the periodicity of the $\mathrm{PC}$ is interrupted, such as the thickness of a layer being changed [3], or another medium being added into the structure [4], some defective modes could be generated within the PBGs. In a simple one-dimensional PC of $(A B)^{n}$, the defect mode can be obtained in a defective structure like $(A B)^{n}(D / B)^{m}(A B)^{n}$, where $D$ is known as a defected layer. The defect mode is a strongly localized defect state which, in general, can be seen in the transmission spectrum with a sharp narrow resonant peak. With the presence of resonant peak in transmittance, the structure is often used as a narrowband transmission filter [5].

The PBG structures can be used in various applications, such as add-drop multiplexers, waveguides, mirrors, and resonant cavities [6-10]. Meanwhile, the optical confinement effect in photonic crystals with defects can be exploited to enhance the nonlinear optical effect. One possible method to realize a nonlinear photonic crystal structure is to insert a nonlinear optical material between two distributed-Bragg reflectors (DBRs), where each DBR consists of alternating high- and low-index layers with quarter-wave thicknesses [11]. Another possible method is to use a nonlinear optical material as a lattice element of the photonic crystal [12].

In this research, we studied the linear and the nonlinear optical properties of a nonlinear photonic crystal: $\left(n_{h} / n_{l}\right)^{4} /\left(N_{d}{ }^{(N L)} / n_{l}\right)^{m} /\left(n_{l} / n_{h}\right)^{4}$. First, we ignored the non-linear nature of the refractive index in the defect layer and investigated the linear optical properties of the photonic crystal. The transmission spectrum was obtained by applying the optical transfer matrix formalism to the photonic crystal. Next, we investigated the transmission spectrum by including the nonlinear defect layer with the order $m=1,2,3$ and studied the shift of the transmission peaks. Then, we studied the transmission variation with the spatial optical intensity distribution inside the nonlinear defect layer.

\section{Linear and Nonlinear Optical Properties of Photonic Crystals}

We consider a one-dimensional photonic crystal structure, as shown in Figure 1, the structure of this photonic crys- 


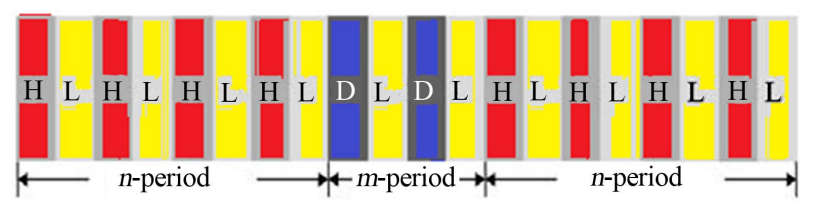

Figure 1. Schematic diagram of 1-D photonic crystal with defect.

tal constituted of high (A) and low(B) refractive index materials with $\mathrm{m}$ layers of defect materials. Here the thickness of $\mathrm{A}$ and $\mathrm{B}$ i.e., $\mathrm{SiO}_{2}$ and $\mathrm{Si}$ is $d_{1}$ and $d_{2}$, corresponding to the materials with refractive indices $n_{1}$ and $n_{2}$, respectively. The concept of defect in the PBG materials has led to a large number of applications as for the semiconductor materials. The suitable thickness of $\mathrm{Ba}-$ $\mathrm{TiO}_{3}$, of $\mathrm{m}$ layers is introduced as the defect layer in the PC. For TE wave, the characteristic matrix for a single period is given by

$$
M=\left[\begin{array}{ll}
M_{11} & M_{12} \\
M_{21} & M_{22}
\end{array}\right]=\prod_{j=1}^{N} M_{j}
$$

If the $j^{\text {th }}$ layer is characterized by the refractive index, $n_{j}$, the extinction coefficient, $k_{j}$, and the thickness, $d_{j}$ then the individual transfer matrix, $M_{j}$, is given by

$$
M_{j}=\left(\begin{array}{cc}
\cos \left(\frac{2 \pi}{\lambda} p_{j} d_{j}\right) & i \frac{1}{p_{j}} \sin \left(\frac{2 \pi}{\lambda} p_{j} d_{j}\right) \\
i p_{j} \sin \left(\frac{2 \pi}{\lambda} p_{j} d_{j}\right) & \cos \left(\frac{2 \pi}{\lambda} p_{j} d_{j}\right)
\end{array}\right)
$$

where $p_{j}=N_{j} \cos \theta_{j}$

$N_{j}=n_{j}+i k_{j}$ is the complex refractive index of the $j^{\text {th }}$ layer, and $\theta_{j}$ is the angle of incidence for the $j^{\text {th }}$ layer. For the wavelength $\lambda=0.633 \mu \mathrm{m}$. The overall structure remains $(H L)^{4}(D L)^{m}(H L)^{4}$, where $H$ and $L$ remain to be $\mathrm{Si}$ and $\mathrm{SiO}_{2}$, respectively. The thickness of $\mathrm{H}$ and $\mathrm{L}$ layers remains to be $50 \mathrm{~nm}$ and the thickness of $D$ layer is $150 \mathrm{~nm}$. The refractive indices for A and B and defect layer are $3.45,1.45$ and 2.416 respectively [13]. Here we analysed the photonic band gap by suitable absorption mechanism. A saturable absorption-based photonic crystal is described by

$$
\left(n_{h} / n_{l}\right)^{4} /\left(N_{d}^{(N L)} / n_{l}\right)^{m} /\left(n_{l} / n_{h}\right)^{4}
$$

where $N_{d}{ }^{(N L)}=n_{d}{ }^{(L)}+i k_{d}^{(N L)}$

$$
\begin{aligned}
& k_{d}{ }^{(N L)}=\frac{k_{d}^{(L)}}{1+b I} \\
& \beta=\frac{\omega}{c} b=\frac{2 \pi}{\lambda} b
\end{aligned}
$$

Here, $I$ is the laser-beam intensity at the sample position, and $\beta$ is the nonlinear absorption coefficient that characterizes the saturable absorption of the nonlinear defect. To compute the defect mode in the transmission spectra, we employ the transfer matrix method (TMM) [14]. The transmission coefficient for tunneling through such as structure is given by

$$
t=\frac{4}{\left(m_{11}+m_{22}\right)^{2}+\left(m_{12}+m_{21}\right)^{2}}
$$

We now turn our attention to the voltage dependence of the defect modes with a nonlinear optical material, namely $\mathrm{BaTiO}_{3}$.

\section{Bias Voltage Dependence of Defect Modes}

When an external voltage is applied to the Defect layer, as depicted in Figure 6 taking $m=2$ for example, its index of refraction will change with the electric field due to the electro-optic effect.

The relation can be expressed as [15]:

$$
n_{B T}^{\prime}=n_{B T}-\frac{1}{2} n_{B T}^{3} \gamma_{B T} E
$$

where $\gamma_{B T}$ is the electro-optic coefficient of the Barium Titanante, with the value of $\gamma_{B T}$ is $820 \times 10^{-12} \mathrm{~m} / \mathrm{V}$, and $E$ is the externally applied electric field. The relation between $E$ and the external applied voltage $V$ is $E=$ $V /(0.4 \mathrm{~mm})$, where $0.4 \mathrm{~mm}$ is the distance between the electrode pair. The index of refraction of Barium Titanate is 2.416 at zero bias voltage. The refractive index of Barium Titanante at various external voltage applied is calculated using the above formula. Hence, the transmittance peak in the photonic band gap is calculated at various voltages.

\section{Results and Discussion}

The photonic crystals consisting of $\mathrm{Si}_{-} \mathrm{Sio}_{2}$ with defect layer is analysed. The schematic diagram of $1 \mathrm{D}$ photonic crystals is shown in Figure 1. Here, we have studied both linear effect and nonlinear effect of the photonic crystal. It was found that a photonic gap exists in the above said photonic crystal without the defect layer as suggested by some recent literature [16]. It is shown in Figure 2. The value of the gap was found between 427 $\mathrm{nm}$ and $630 \mathrm{~nm}$. We also found that when we introduce a defect layer $\mathrm{BaTio}_{3} / \mathrm{Sio}_{2}$ in the above said photonic crystals, an additional mode of propagation is obtained in the photonic band gap. The photonic band gap is between $420 \mathrm{~nm}$ and $665 \mathrm{~nm}$. It can be seen that there is a sharp transmission peak at $552 \mathrm{~nm}$ in the gap as shown in Figure 3. The transmission peak is caused by the defect state. When the order of the defect layer increases such as $\mathrm{m}=$ 2, 3, 4 etc, additional modes occurs in photonic band gap as shown in Figures $\mathbf{4}$ and $\mathbf{5}$. When the number of defect layer increases one of the transmittance peak goes to higher values. It almost reaches the maximum value of 1 . The variation of maximum value of the transmittance 


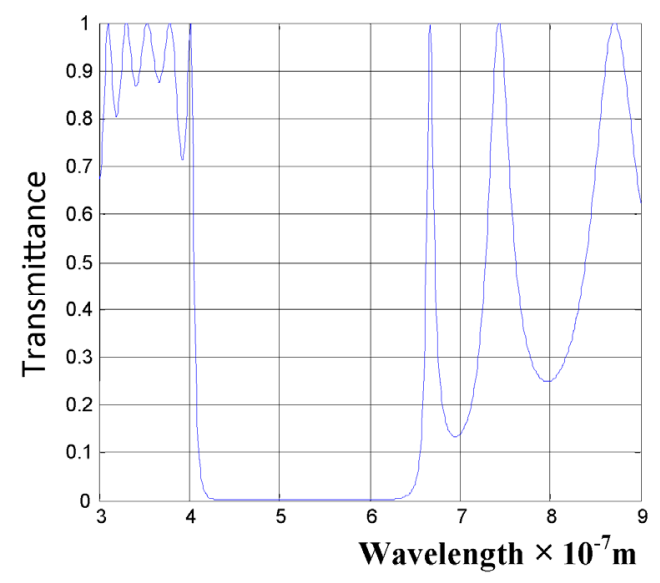

Figure 2. PBG crystal without defect.

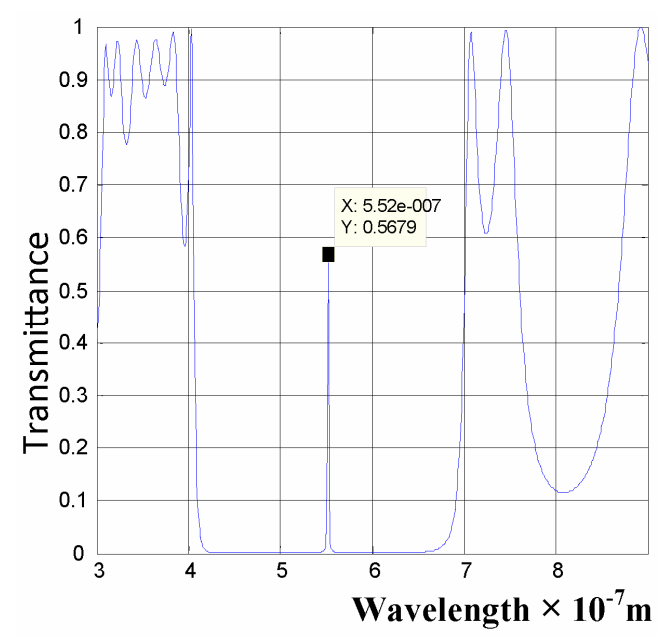

Figure 3. PBG crystal with defect for the value $m=1$.

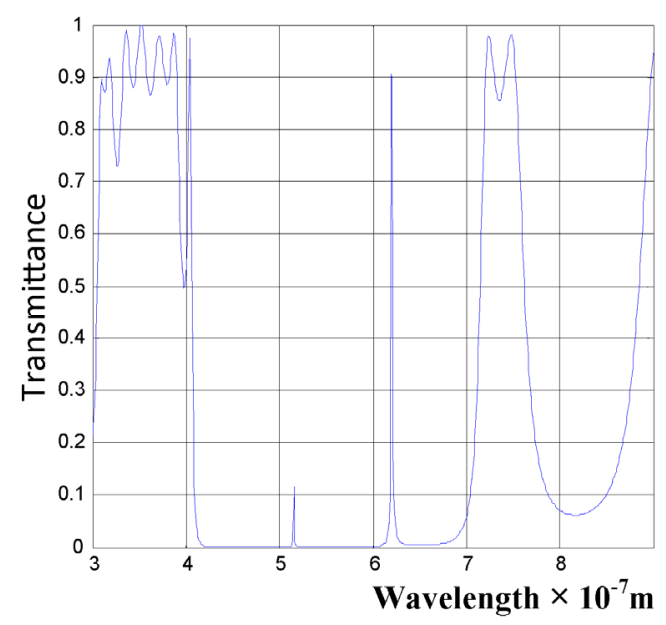

Figure 4. PBG crystal with defect for the value $m=2$.

peak with number of defect layers is plotted in Figure 6. This value of transmittance peak occurs at various wavelengths with the number of defect layers as shown in Figure 7. It is also observed there is no such variation

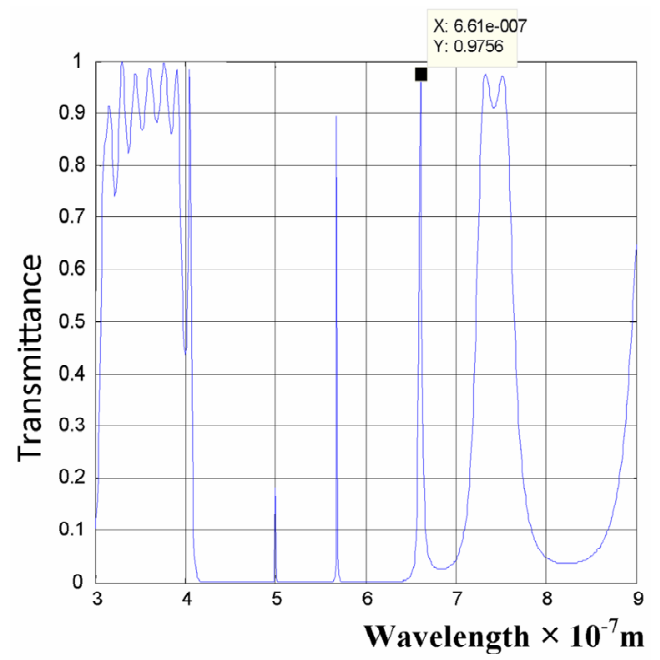

Figure 5. PBG crystal with defect for the value $m=3$.

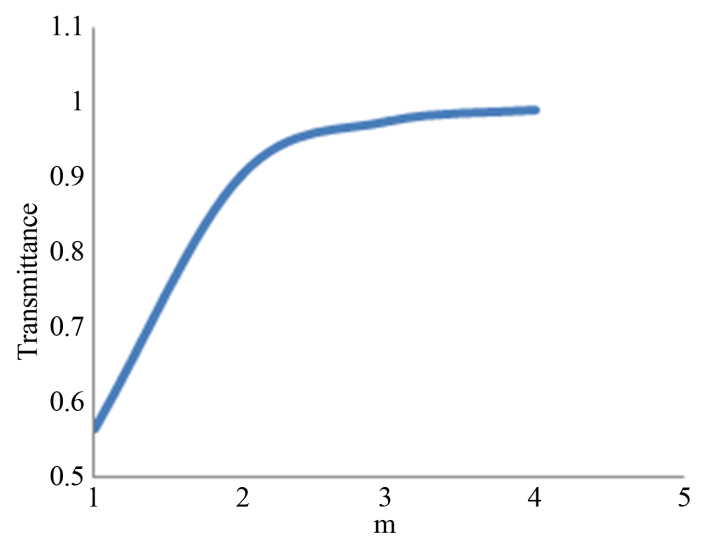

Figure 6. Variation of transmittance with defect states.

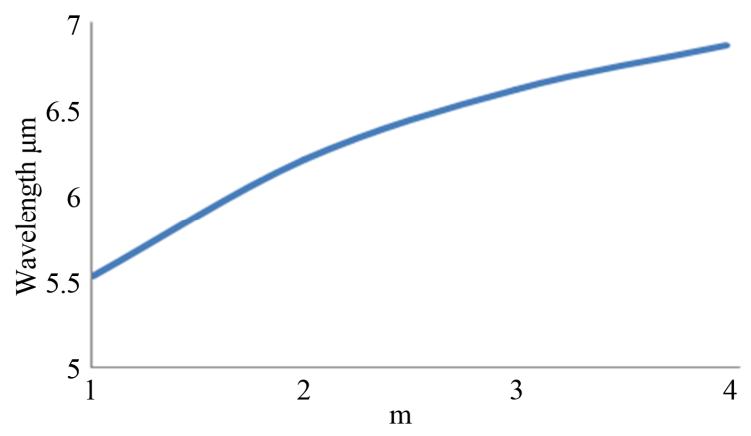

Figure 7. The variation of the wavelength corresponding to the transmittance peak with defect states.

occurs in the photonic band gap width. When the nonlinear effect is included, we observed a slight variation in the transmittance peak. For $m=1$ with a structure of $\left(n_{h} / n_{l}\right)^{4} /\left(N_{d}(N L) / n_{l}\right)^{m} /\left(n_{l} / n_{h}\right)^{4}$, the voltage dependence of the transmittance peak in the PBG is analysed. The wavelengths of peaks are summarized in Table 1. 
Table 1. The wavelengths of peaks for $\left(n_{h} / n_{l}\right)^{4} /\left(N_{d}^{(N L)} / n_{l}\right){ }^{1 /}$ $\left(\mathbf{n}_{1} / \mathbf{n}_{h}\right)^{4}$.

\begin{tabular}{cc}
\hline External applied bias $(\mathrm{kV})$ & Wavelength of the transmittance peaks \\
\hline-30 & $610 \mathrm{~nm}$ \\
0 & $552 \mathrm{~nm}$ \\
30 & $490 \mathrm{~nm}$ \\
\hline
\end{tabular}

\section{Conclusion}

The linear and nonlinear optical properties of nonlinear photonic crystal are studied. The various transmittance peak occurrence is analyzed with the defect states. The defect states in the photonic crystal introduce additional modes in the photonic band gap. When the applied voltage increases the wavelength at which the maximum transmittance peak occurrence decreases. This can be greatly applied in many fields such as diode laser, nonthreshold laser, dense wavelength division multiplex and $\mathrm{PC}$ fiber as well as the multichannel transmission filter.

\section{Acknowledgements}

We gratefully acknowledge University Grants Commission, India (Ref: No. F. 41-977/2012(SR)) for the Financial Support of this work.

\section{REFERENCES}

[1] E. Yablonovitch, "Inhibited Spontaneous Emission in SolidState Physics and Electronics," Physical Review Letters, Vol. 58, 20, 1987, pp. 2059-2062. http://dx.doi.org/10.1103/PhysRevLett.58.2059

[2] S. John, "Strong Localization of Photons in Certain Disordered Dielectric Superlattices," Physical Review Letters, Vol. 58, No. 23, 1987, pp. 2486-2489. http://dx.doi.org/10.1103/PhysRevLett.58.2486

[3] G. Boedecker and C. Henkel, "All-Frequency Effective Medium Theory of a Photonic Crystal," Optics Express, Vol. 11, No. 13, 2003, pp. 1590-1595. http://dx.doi.org/10.1364/OE.11.001590

[4] Y. Akahane, T. Asano, B. S. Song and S. Noda, "AllFrequency Effective Medium Theory of a Photonic Crystal," Nature, Vol. 425, No. 6961, 2003, pp. 885-989. http://dx.doi.org/10.1038/nature02063

[5] S. J. Orfanidis, "Electromagnetic Waves and Antennas," Ch. 6, Rutgers University, Rutgers, 2008. www.ece.rutgers.edu/ orfanidi/ewa

[6] M. Imada, S. Noda, A. Chutinan, M. Mochizuki and T. Tanaka, "Channel Drop Filter Using a Single Defect in a 2-D Photonic Crystal Slab Waveguide," Journal of Light- wave Technology, Vol. 20, No. 5, 2002, pp. 873-878. http://dx.doi.org/10.1109/JLT.2002.1007943

[7] D. R. Solli, C. F. McCormick and J. M. Hickmann, "Polarization-Dependent Reflective Dispersion Relations of Photonic Crystals for Waveplate Mirror Construction," Journal of Lightwave Technology, Vol. 24, No. 10, 2006, pp. 3864-3867. http://dx.doi.org/10.1109/JLT.2006.882252

[8] S. Y. Lin, E. Chow, V. Hietala, P. Villeneuve and J. Joannopoulos, "Experimental Demonstration of Guiding and Bending of Electromagnetic Waves in a Photonic Crystal," Science, Vol. 282, No. 5387, 1998, pp. 274-276. http://dx.doi.org/10.1126/science.282.5387.274

[9] S. Noda, A. Chutinan and M. Imada, "Trapping and Emission of Photons by a Single Defect in a Photonic Bandgap Structure," Nature, Vol. 407, No. 6804, 2000, pp. 545-658. http://dx.doi.org/10.1038/35036532

[10] O. Painter, R. K. Lee, A. Scherer, A. Yariv, J. D. Obrien, P. D. Dapkus and I. Kim, "Two-Dimensional Photonic Band-Gap Defect Mode Laser," Science, Vol. 284, No. 5421, 1999, pp. 1819-1821. http://dx.doi.org/10.1126/science.284.5421.1819

[11] J. E. Shen, Z. Zhang, Z. Hua, G. Ma and S. H. Tang, "Observation of Two-Photon Absorption Enhancement at Double Defect Modes in One-Dimensional Photonic Crystals," Applied Physics Letters, Vol. 88, No. 1, 2006, Article ID: 011113. http://dx.doi.org/10.1063/1.2162698

[12] I. V. Guryev, O. V. Shulika, I. A. Sukhoivanov and O. Mashoshina, "Improvement of Characterization Accuracy of the Nonlinear Photonic Crystals Using Finite Elements-Iterative Method," Applied Physics B, Vol. 84, No. 1-2, 2006, pp. 83-87. http://dx.doi.org/10.1007/s00340-006-2330-y

[13] E. D. Palik, "Handbook of Optical Constants and Solids," Academic, Orlando, 1985.

[14] M. Born and E. Wolf, "Principles of Optics," 6th Edition, Pergamon, Oxfors, 1980.

[15] Q. Zhu and Y. Zhang, "Defect Modes and Wavelength Tuning of One-Dimensional Photonic Crystal with Lithium Niobate," Optik, Vol. 120, No. 4, 2009, pp. 195-198. http://dx.doi.org/10.1016/i.ijleo.2007.06.024

[16] G. J. Lee, Y.P. Lee, H. Y. Kim, S. Kim and I. Park, "Detailed Treatment of the Nonlinear Optical Properties of Nonlinear Photonic Crystals," Journal of the Korean Physical Society, Vol. 55, No. 3, 2009, pp. 1237-1242. http://dx.doi.org/10.3938/jkps.55.1237 